\title{
Statistical Entropy of the Schwarzschild black hole
}

\author{
Mariano Cadon* \\ Dipartimento di Fisica, Università di Cagliari, and INFN sezione di Cagliari, \\ Cittadella Universitaria 09042 Monserrato, ITALY
}

\begin{abstract}
We derive the statistical entropy of the Schwarzschild black hole by considering the asymptotic symmetry algebra near the $\mathcal{I}^{-}$boundary of the spacetime at past null infinity. Using a two-dimensional description and the Weyl invariance of black hole thermodynamics this symmetry algebra can be mapped into the Virasoro algebra generating asymptotic symmetries of anti-de Sitter spacetime. Using lagrangian methods we identify the stress-energy tensor of the boundary conformal field theory and we calculate the central charge of the Virasoro algebra. The Bekenstein-Hawking result for the black hole entropy is regained using Cardy's formula. Our result strongly supports a non-local realization of the holographic principle
\end{abstract}

Black holes can be understood as thermodynamical systems with characteristic temperature and entropy [1, 2]. In the last decade a lot of effort has been devoted to understand the microscopic origin of black hole thermodynamics. A detailed microscopical explanation of the thermodynamical properties of black holes would represent not only an important tool for understanding the quantum behavior of gravity but also a way to give a fundamental meaning to the holographic principle 3, 4]. A variety of approaches have been used to explain the microscopical origin of the Bekenstein-Hawking entropy: string theoretical (D-Brane) approaches 5, 6, 7, 8, 9], methods based on loop quantum gravity [10], induced gravity [1, 12], asymptotic symmetries 13, 14, 15, 16, 17, 18, 19, 20, 21, 22, 23. and canonical quantization [24]. None of these derivations can be considered as completely satisfactory. In some cases the computation works only for a restrict class of solutions, e.g supersymmetric or asymptotically Anti-de Sitter (AdS) solutions. In other cases the origin of the microscopic degrees of freedom responsible for the black hole entropy is completely obscure. Nonetheless, the fact that completely independent methods give more or less the same (right) answer strongly indicates that the computation of the statistical black hole entropy can be performed without detailed knowledge of the physics governing the microscopical degrees of freedom.

If this is the case the statistical black hole entropy should be explained in terms of fundamental features (e.g symmetries) of the "emergent" classical theory of gravity of which black holes are solutions. The most natural realization of this scenario is represented by near-horizon conformal symmetries controlling the black hole entropy trough Cardy's formula for the density of states [21, 23]. Carlip has pointed out the main ingredients to be used in this approach: a) Near-horizon conformal symmetries, b) Asymptotic symmetries, c) Canonical realization of the symmetries, $d$ ) Horizon constraints [21]. However, the approach of Carlip has some drawbacks. To avoid the complications of the Hamiltonian formulation with a null slicing one has to consider a distorted, "almost" null, horizon [21, 23]. The null limit of this formulation is usually singular and the null normal does not have a unique normalization. Moreover, the result seems to depend on the horizon boundary conditions [25, 26] and the constraint algebra is complicated by the appearance of additional constraints related to the use of a null frame in the slicing [23].

Apart from these technical difficulties, derivations that use near-horizon symmetries leave unanswered the question about the localization of the microstates responsible for the black hole entropy. A number of cases are known for which the Bekenstein-Hawking entropy can be reproduced using a conformal filed theory (CFT) living either on the black hole horizon or on a timelike asymptotical boundary of the spacetime 13, 14, 15, 16, 17]. An answer to this question is of fundamental importance for understanding if the holographic principle is realized in a local or non-local way.

In this letter we will avoid the subtleties of the canonical approach by considering a purely lagrangian formulation of the gravity theory. We will derive the statistical entropy of the Schwarzschild black hole by considering the asymptotic symmetry algebra near the $\mathcal{I}^{-}$boundary of the spacetime at past null infinity. Using a two-dimensional (2D) model to describe the radial modes of Einstein gravity and the Weyl invariance of black hole thermodynamics [27], this symmetry algebra is mapped in the Virasoro algebra generating asymptotic symmetries of 2D AdS spacetime. Using lagrangian methods, we identify the stress-energy tensor for the boundary conformal field theory and we calculate the central charge of the Virasoro algebra. The Bekenstein-Hawking result for the black hole entropy is obtained from Cardy's formula.

The Schwarzschild black hole of general relativity behaves as a thermodynamical system with temperature $T$ and entropy $S$ given by

$$
T=\frac{1}{8 \pi G M}, \quad S=4 \pi G M^{2},
$$


where $M$ is the black hole mass and $G$ is Newton constant. The black hole admits a two-dimensional effective description. The effective 2D gravity model can be obtained from the four-dimensional (4D) Einstein action retaining only the radial modes of the gravitational field,

$$
d s_{(4)}=d s_{(2)}+\frac{2}{\lambda^{2}} \phi d \Omega_{2}^{2},
$$

where $\lambda=1 / \sqrt{G}$ is the Planck mass (we use natural units) and $\phi$ is a scalar field parametrizing the radius of the transverse 2-Sphere. Because the causal structure and the thermodynamical parameters (mass $M$, temperature $T$ and entropy $S$ ) of the 2D solutions are invariant under Weyl rescaling of the 2D metric [27], the Schwarzschild solution can be described by an equivalence class (under conformal transformations of the metric) of 2D gravity models. Let us use this freedom to pick up a 2D gravity model with asymptotic anti-de Sitter behavior.

Performing the dimensional reduction (2) in the 4D Einstein-Hilbert action and the Weyl rescaling of the 2D metric $g_{\mu \nu}^{(2)} \rightarrow(2 \phi) g_{\mu \nu}^{(2)}$, we end up with the 2D dilaton gravity model,

$$
S=\frac{1}{2} \int d^{2} x \sqrt{-g}\left(\phi R+\frac{3}{2} \frac{(\nabla \phi)^{2}}{\phi}+2 \lambda^{2} \phi\right) .
$$

The static solutions of the gravity model (3) are

$$
d s^{2}=-\left[(\lambda x)^{2}-\frac{2 M}{\lambda}(\lambda x)^{3}\right] d t^{2}+\left[(\lambda x)^{2}-\frac{2 M}{\lambda}(\lambda x)^{3}\right]^{-1} d x^{2}, \quad \phi=\frac{1}{2}(\lambda x)^{-2} .
$$

One can easily check that the solution (4) describes the 2D sections of the 4D Schwarzschild black hole after performing the Weyl rescaling $\hat{g}_{\mu \nu}=(2 \phi) g_{\mu \nu}$ and changing the radial coordinate $\lambda x=1 /(\lambda r)$. Using well-known formulas, one can easily calculate the $2 \mathrm{D}$ thermodynamical parameters associated with solution (4), reproducing exactly those of the Schwarzschild black hole (1).

Solution (4) is asymptotically AdS but not in the usual sense of its $x \rightarrow \infty$ behavior (For a discussion of 2D AdS spacetime $\left(\mathrm{AdS}_{2}\right)$ and its different parametrization see Ref. [28])). It behaves as $\mathrm{AdS}_{2}$ for $x \rightarrow 0$, when it takes the form $d s^{2}=-(\lambda x)^{2} d t^{2}+(\lambda x)^{-2} d x^{2}$. The exterior region of the Schwarzschild black hole, $2 M G<r<\infty$, is mapped by the a Weyl transformation and coordinate transformation into the region $0<x<1 /(2 M)$ of the 2D solution (44). The corresponding Penrose diagrams of the solutions are also the same in the considered range of the coordinates $r, x$. They have both the shape of a diamond, with the $\mathcal{I}^{ \pm}, r \rightarrow \infty$ null boundaries (past and future horizon $\mathcal{H}^{ \pm}$ at $r=2 G M$ ) of the Schwarzschild spacetime mapped into the internal $I^{ \pm}, x=0$ null boundaries (past and future horizon $H^{ \pm}$at $x=1 /(2 M)$ ) of the spacetime (4).

To discuss the behavior of the 2D gravity model on the null boundaries $I^{ \pm}$we need to write the solution (4) using light-cone coordinates,

$$
d s^{2}=2 g_{+-} d x^{+} d x^{-}=-\left((\lambda x)^{2}-\frac{2 M}{\lambda}(\lambda x)^{3}\right) d x^{+} d x^{-}, \quad \phi=\frac{1}{2}(\lambda x)^{-2},
$$

where $x^{ \pm}$are implicitly defined by $(\lambda / 2)\left(x^{+}-x^{-}\right)=(\lambda x)^{-1}+(2 M / \lambda)[\ln (1-2 M x)-\ln (\lambda x)], \quad t=(1 / 2)\left(x^{+}+x^{-}\right)$.

The symmetry relevant for our discussion is the group of the asymptotic symmetries (ASG) of the Schwarzschild spacetime near $\mathcal{I}^{ \pm}$. A discussion of the ASG for asymptotically flat spacetimes can be found in Ref. [29]. The previously discussed Weyl transformation maps the $\mathcal{I}^{ \pm}$boundary of the Schwarzschild spacetime into the $I^{ \pm}$inner boundary of our 2D spacetime, hence we can equivalently consider the ASG of our model (3) for $x \rightarrow 0$. Our 2D spacetime behaves for $x \rightarrow 0$ as $\mathrm{AdS}_{2}$, we therefore expect the ASG to be the conformal group in one dimension.

The $x=0$ inner boundary of the spacetime splits in the two orthogonal parts $I^{+}$and $I^{-}$. When discussing the ASG we are forced to consider only one of those two parts. We will choose $I^{-}$, that is we discuss the asymptotic behavior of the solutions for $x^{-} \rightarrow-\infty$ and $0<x^{+}<\infty$. For $x^{-} \rightarrow-\infty$ solution (5) behaves as

$$
d s^{2}=-\left[\frac{4}{\left(\lambda x^{-}\right)^{2}}+O\left(\frac{\ln \left(-\lambda x^{-}\right)}{\left(x^{-}\right)^{3}}\right)\right] d x^{+} d x^{-}, \quad \phi=\frac{\left(\lambda x^{-}\right)^{2}}{8}+O\left(x^{-} \ln \left(-\lambda x^{-}\right)\right) .
$$

We are therefore led to impose the following boundary conditions at $x^{-} \rightarrow-\infty$

$$
\begin{aligned}
& g_{+-}=-\frac{2}{\left(\lambda x^{-}\right)^{2}}+O\left(\frac{\ln \left(-\lambda x^{-}\right)}{\left(x^{-}\right)^{3}}\right), \quad g_{++}=O\left(\frac{1}{x^{-}}\right), \\
& g_{--}=0 \text { (identically), } \phi=\frac{\left(\lambda x^{-}\right)^{2}}{8}+O\left(x^{-} \ln \left(-\lambda x^{-}\right)\right) .
\end{aligned}
$$


The asymptotic form (7) is preserved by infinitesimal diffeomorphisms $\chi^{\mu}\left(x^{-}, x^{+}\right)$given by

$$
\chi^{+}=\varepsilon\left(x^{+}\right), \quad \chi^{-}=\dot{\varepsilon}\left(x^{+}\right) x^{-}+O(1),
$$

where the dot denotes derivation with respect to $x^{+}$. Expanding $\varepsilon\left(x^{+}\right)$in Laurent series yields the generators of the ASG,

$$
L_{k}=\left[(k+1) x^{-}\left(\lambda x^{+}\right)^{k}+O(1)\right] \partial_{-}+\frac{1}{\lambda}\left[\left(\lambda x^{+}\right)^{k+1}+O\left(\frac{1}{x^{-}}\right)\right] \partial_{+},
$$

which satisfy (after the sign flipping $L_{k} \rightarrow-L_{k}$ ) the Virasoro algebra

$$
\left[L_{k}, L_{m}\right]=(k-m) L_{k+m}+\frac{c}{12} k^{3} \delta_{k+m 0},
$$

where we have taken into account the possibility of a central extension $c$.

The boundary conditions (77) allows us to define boundary fields $\Gamma_{0}\left(x^{+}\right), \Gamma_{1}\left(x^{+}\right) \ldots, \hat{\Gamma}_{0}\left(x^{+}\right), \hat{\Gamma}_{1}\left(x^{+}\right) \ldots$, $\gamma_{0}\left(x^{+}\right), \gamma_{1}\left(x^{+}\right), \ldots$, which describe deformations of the boundary $I^{-}$and of the scalar field $\phi$,

$$
\begin{aligned}
g_{+-} & =-\frac{1}{2}\left[\frac{1}{X^{2}}+\Gamma_{0} \frac{\ln (-X)}{X^{3}}+\frac{\Gamma_{1}}{X^{3}}+\Gamma_{2} \frac{\ln ^{2}(-X)}{X^{4}}+\ldots\right] \\
g_{++} & =\frac{\hat{\Gamma}_{0}}{X}+\hat{\Gamma}_{1} \frac{\ln (-X)}{X^{2}}+\ldots, \\
\phi & =\gamma_{0} X^{2}+\gamma_{1} X \ln (-X)+\gamma_{2} X+\gamma_{3} \ln ^{2}(-X)+\gamma_{4} \ln (-X)+\gamma_{5}+\ldots,
\end{aligned}
$$

where $X=\left(\lambda x^{-} / 2\right)$.

It is important to notice that the leading terms for the metric and the scalar $\phi$ in the expansion (11) ( hence also in Eq. (7)) are not solution of the classical field equation coming from the action (3). The classical black hole solutions can be recovered only choosing a particular form of the boundary fields in Eqs. (111). This means that we are using a notion of asymptotic symmetry which is slightly different form the usual one (see Ref. [30]). In the usual formulation the leading term in the boundary conditions represents a (background) classical solution that remains invariant under the action of the ASG. In our formulation what is invariant under transformation of the ASG is the leading term in the asymptotic form of the spacetime metric, which does not need to be a solution of the field equations.

The boundary fields $\Gamma_{i}, \hat{\Gamma}_{i}, \gamma_{i}, i=0,1 \ldots$ transform under the action of the ASG as conformal fields of definite weight ( with possible anomalous terms). We have for instance $\delta \Gamma_{0}=\varepsilon \dot{\Gamma}_{0}-\Gamma_{0} \dot{\varepsilon}, \delta \gamma_{0}=\varepsilon \dot{\gamma}_{0}+2 \Gamma_{0} \dot{\varepsilon}$,. In the following we will need the transformation law for the boundary field $\gamma_{5}$,

$$
\delta \gamma_{5}=\varepsilon \dot{\gamma}_{5}+\dot{\varepsilon} \gamma_{4}
$$

The next step in our derivation is to define the charges associated with the generators of the ASG $L_{k}$. To define the charges one usually considers a canonical realization of the ASG [30]. The Hamiltonian approach works well when dealing with timelike (also spacelike, see Ref. [31]) boundaries, is problematic for null boundaries [21, 23]. In fact, foliating the 2D spacetime using null curves one has ambiguities connected with the use of null vectors. On the other hand, if one uses a distorted boundary and a corresponding foliation with spacelike curves, one usually has a singular limit when the boundary becomes null. To avoid these problems we will use a purely Lagrangian method to define the charges associated with the ASG generators.

The covariantly conserved current $J_{\mu}$ associated with an isometry of the spacetime generated by a Killing vector $\chi^{\nu}$ can be written has $J_{\mu}=T_{\mu \nu} \chi^{\nu}$, where $T_{\mu \nu}=(2 / \sqrt{-g})\left(\delta S / \delta g^{\mu \nu}\right)$ is the stress-energy tensor. In two spacetime dimensions one can always integrate, locally, the equation $\nabla^{\mu} J_{\mu}=0$, yielding $J_{\mu}=\epsilon_{\mu}^{\nu} \partial_{\nu} \Omega$, where $\Omega$ is a scalar, which can be considered as the charge associated with the isometry generated by $\chi^{\nu}$,

$$
\epsilon_{\mu}^{\nu} \partial_{\nu} \Omega=T_{\mu \nu} \chi^{\nu}
$$

The classical equations of motion give $T_{\mu \nu}=0$, so that on-shell the charges are constant. This definition can be easily generalized to define the charges associated with the generators of the group of asymptotic symmetries. Using light-cone coordinates Eq. (13) yields $\partial_{+} \Omega=-\left(T_{++} \chi^{+}+T_{+-} \chi^{-}\right), \partial_{-} \Omega=T_{--} \chi^{-}+T_{-+} \chi^{+}$. Pushing these equations 
on the $I^{-}$boundary by taking the $x^{-} \rightarrow-\infty$ limit, the second equation becomes an identity, whereas the first yields the definition of the charges associated with the ASG generated by the killing vectors (8),

$$
\Omega(\varepsilon)=-\lim _{x^{-} \rightarrow-\infty} \int_{I^{-}} d x^{+}\left(\varepsilon T_{++}+\dot{\varepsilon} x^{-} T_{+-}\right) .
$$

The term proportional to $x^{-} T_{+-}$vanishes on the $I^{-}$boundary and we have $\Omega(\varepsilon)=\int d x^{+} \varepsilon T$, where $T=$ $-\lim _{x^{-} \rightarrow-\infty} T_{++} . T$ has to be considered as the stress-energy tensor for a one-dimensional CFT living on $I^{-}$. The tensor $T$ can be derived from the action (3) and using Eqs. (11). $T$ is identically zero when the boundary fields (11) are on-shell, i.e when they describe a classical black hole solution of the action (3). For generic off-shell deformations $T$ is the sum of terms, which diverge for $x^{-} \rightarrow-\infty$ (the leading divergence is $\left.\propto\left(x^{-}\right)^{2}\right)$, and finite terms. The simplest way to eliminate these divergences is to consider all deformations (11) on-shell except $\gamma_{5}$. Using this renormalization prescription we get

$$
T=\ddot{\gamma}_{5}
$$

This procedure has a simple physical interpretation. We start from background boundary deformations $\Gamma_{i}^{(b)}, \gamma_{i}^{(b)}$ corresponding to classical black hole solution of a given mass $M$ and allow only for off-shell boundary deformations of the classical configuration that produce charges, which are finite for $x^{-} \rightarrow-\infty$.

The transformation law of $T$ under the action of the ASG can be easily derived using Eq. (12). We have

$$
\delta T=\varepsilon \dot{T}+2 \dot{\varepsilon} T+C\left(\varepsilon, \gamma_{4}, \gamma_{5}\right),
$$

where $C=\gamma_{4} \dddot{\varepsilon}+\ddot{\gamma}_{4} \dot{\varepsilon}+\left(\dot{\gamma}_{5}+2 \dot{\gamma}_{4}\right) \ddot{\varepsilon}$. As expected Eq. (16) is the transformation law of a stress-energy tensor of a CFT living on the $I^{-}$boundary. The anomalous term $C$ has to be evaluated on the classical background configuration, $\gamma_{4}^{(b)}=(2 M / \lambda)^{2}-M x^{+}, \gamma_{5}^{(b)}=(2 M / \lambda)^{2}-M x^{+}+\left(\lambda^{2} / 8\right)\left(x^{+}\right)^{2}$. For macroscopic black holes $(M / \lambda)>>1$. Retaining only the leading term for $(M / \lambda)>>1, C$ takes its standard form, $C=\left(\frac{2 M}{\lambda}\right)^{2} \dddot{\varepsilon}$. From this equation one can read off the value of the central charge of the boundary CFT,

$$
c=48 \frac{M^{2}}{\lambda^{2}} .
$$

Apart from the divergence for $x^{-} \rightarrow \infty$, the charges $\Omega$ are also infrared divergent, owing to the non-compactness of the $I^{-}$boundary. As a further consequence of this divergence the inner product on $I^{-}$is ill-defined preventing a realization of the algebra (10) trough the charges (14). Both problems can be solved introducing an infrared cutoff $R$ and compactifying the $I^{-}$boundary.

The size $R$ of compactified $I^{-}$is set by the temperature (1) of the Schwarzschild black hole. $R$ has to be identified in terms of the periodicity $\beta=1 / T$ of the euclidean time: $R=(1 / 2)(\beta / 2 \pi)=2 M / \lambda^{2}($ the $1 / 2$ factor takes into account the presence of the orthogonal $I^{+}$boundary). The charges are now finite and become,

$$
\Omega(\varepsilon)=\int_{0}^{R} d x^{+} \varepsilon T .
$$

Using this expression one can easily calculate the charge ( the eigenvalue) of the Virasoro operator $L_{0}$. Reading in Eq. (9) the value of $\varepsilon$ corresponding to the $L_{0}$ operator, $\left(\varepsilon=x^{+}\right)$and using Eq. (15) evaluated on-shell into Eq. (18), one gets

$$
\Omega\left(L_{0}\right)=\frac{M^{2}}{2 \lambda^{2}} .
$$

The Virasoro algebra (10) and in particular the central charge $c$ are determined by the short distance behavior of the theory and are independent of both the infrared cutoff $R$ and the topology of the $I^{-}$boundary. We can therefore give a realization of the Virasoro algebra (10) in terms of the charges (18) by considering a (infrared regularized) boundary with the topology of $S^{1}$ and writing the transformation law of $\Omega(\varepsilon)$ in operatorial form,

$$
\delta_{\xi} \Omega(\varepsilon)=i[\Omega(\varepsilon), \Omega(\xi)],
$$

where $\varepsilon, \xi$ parametrize two infinitesimal ASG transformations. Expanding in Fourier modes $\Omega(\varepsilon)=\sum_{m} a_{m} L_{m}$, $\varepsilon=\left[R /(2 \pi)^{3 / 2}\right] \sum_{m} a_{m} f_{m}, T=(2 \pi / R)^{2} \sum_{m} L_{m} f_{-m}$, with $f_{m}=\exp \left(2 \pi i m x^{+} / R\right)$ and using Eqs. (16), (18) into (20), one finds that the operators $L_{m}$ satisfy the algebra (10) with central charge given exactly by Eq. (17). 
The entropy associated with the boundary CFT characterized by eigenvalue $l_{0}$ of the operator $L_{0}$ and central charge $c$, is given in the semiclassical regime we are considering by the Cardy formula $S=2 \pi \sqrt{c l_{0} / 6}$. Using Eqs. (17) and (19) we get for the entropy,

$$
S=4 \pi \frac{M^{2}}{\lambda^{2}},
$$

matching exactly the Bekenstein-Hawking entropy of the Schwarzschild black hole.

The main result of this paper is that the Bekenstein-Hawking entropy of the Schwarzschild black hole can be correctly reproduced by counting states of a CFT defined on the $\mathcal{I}^{-}$boundary of the spacetime at past null infinity. Because the same result can be obtained using a CFT defined on the black hole horizon, this seems to imply a non-local realization of the holographic principle. Our derivation method uses a purely lagrangian formulation of the gravity theory and it is therefore free of the usual problems affecting the Hamiltonian formulation in null frames. The divergences of the boundary stress-energy tensor for $x^{-} \rightarrow-\infty$ can be removed in a non-ambiguous way. The only source of ambiguity comes from the infrared divergence due to the infinite length of the $I^{-}$boundary. This divergence has been removed by compactification. The radius of the compactified boundary has been fixed in a natural way in terms of inverse temperature of the black hole.

I am grateful to N. Pinamonti for discussions about the ambiguities of the canonical formulation in the presence of null boundaries

* Electronic address: mariano.cadoni@ca.infn.it

[1] J. D. Bekenstein, Lett. Nuovo Cim. 4 (1972) 737.

[2] S. W. Hawking, Commun. Math. Phys. 43 (1975) 1999.

[3] L. Susskind, J. Math. Phys. 36 (1995) 6377 arXiv:hep-th/9409089.

[4] R. Bousso, Rev. Mod. Phys. 74 (2002) 825 arXiv:hep-th/0203101.

[5] A. Strominger and C. Vafa, Phys. Lett. B 379 (1996) 99 arXiv:hep-th/9601029.

[6] G. T. Horowitz and A. Strominger, Phys. Rev. Lett. 77 (1996) 2368 arXiv:hep-th/9602051.

[7] C. V. Johnson, R. R. Khuri and R. C. Myers, Phys. Lett. B 378 (1996) 78 arXiv:hep-th/9603061].

[8] C. G. . Callan and J. M. Maldacena, Nucl. Phys. B 472 (1996) 591 arXiv:hep-th/9602043.

[9] O. Aharony, S. S. Gubser, J. M. Maldacena, H. Ooguri and Y. Oz, Phys. Rept. 323 (2000) 183 arXiv:hep-th/9905111.

[10] A. Ashtekar, J. Baez, A. Corichi and K. Krasnov, Phys. Rev. Lett. 80 (1998) 904 arXiv:gr-qc/9710007.

[11] V. P. Frolov and D. V. Fursaev, Phys. Rev. D 56 (1997) 2212 arXiv:hep-th/9703178.

[12] D. V. Fursaev, Phys. Part. Nucl. 36 (2005) 81 [Fiz. Elem. Chast. Atom. Yadra 36 (2005) 146] arXiv:gr-qc/0404038.

[13] A. Strominger, JHEP 9802 (1998) 009 arXiv:hep-th/9712251.

[14] M. Cadoni and S. Mignemi, Phys. Rev. D 59 (1999) 081501 arXiv:hep-th/9810251].

[15] M. Cadoni and S. Mignemi, Nucl. Phys. B 557 (1999) 165 arXiv:hep-th/9902040.

[16] S. Carlip, Phys. Rev. Lett. 82 (1999) 2828 arXiv:hep-th/9812013.

[17] M. Caldarelli, G. Catelani and L. Vanzo, JHEP 0010 (2000) 005 arXiv:hep-th/0008058.

[18] D. J. Navarro, J. Navarro-Salas and P. Navarro, Nucl. Phys. B 580 (2000) 311 arXiv:hep-th/9911091.

[19] J. l. Jing and M. L. Yan, Phys. Rev. D 63 (2001) 024003 arXiv:gr-qc/0005105.

[20] M. I. Park, Nucl. Phys. B 634 (2002) 339 arXiv:hep-th/0111224.

[21] S. Carlip, Phys. Rev. Lett. 88 (2002) 241301 arXiv:gr-qc/0203001.

[22] A. J. M. Medved, Class. Quant. Grav. 19 (2002) 2503 arXiv:hep-th/0201079.

[23] S. Carlip, Class. Quant. Grav. 22 (2005) 1303 arXiv:hep-th/0408123.

[24] V. Moretti and N. Pinamonti, arXiv:gr-qc/0507050

[25] N. Pinamonti and L. Vanzo, Phys. Rev. D 69 (2004) 084012 arXiv:hep-th/0312065.

[26] G. Kang, J. i. Koga and M. I. Park, Phys. Rev. D 70 (2004) 024005 arXiv:hep-th/0402113.

[27] M. Cadoni, Phys. Lett. B 395 (1997) 10 arXiv:hep-th/9610201.

[28] M. Cadoni and S. Mignemi, Phys. Rev. D 51 (1995) 4319 arXiv:hep-th/9410041.

[29] C. Dappiaggi, JHEP 0411 (2004) 011 arXiv:hep-th/0410026.

[30] J. D. Brown and M. Henneaux, Commun. Math. Phys. 104 (1986) 207.

[31] M. Cadoni, P. Carta, M. Cavaglia and S. Mignemi, Phys. Rev. D 66 (2002) 065008 arXiv:hep-th/0205211. 